\title{
Do hyperoxaemia and hypocapnia add to the risk of brain injury after intrapartum asphyxia?
}

\author{
G Klinger, J Beyene, P Shah, M Perlman
}

Arch Dis Child Fetal Neonatal Ed 2005;90:F49-F52. doi: 10.1136/adc.2003.048785

See end of article for authors' affiliations

.....................

Correspondence to: Professor Perlman, The Hospital for Sick Children and University of Toronto, 555 University Avenue, Toronto M5G 1X8, Ontario, Canada; max. perlman@sympatico.ca

Accepted 23 July 2004

\begin{abstract}
Background: Episodes of hyperoxaemia and hypocapnia, which may contribute to brain injury, occur unintentionally in severely asphyxiated neonates in the first postnatal hours.

Objective: To determine whether hyperoxaemia and/or hypocapnia during the first 2 hours of life add to the risk of brain injury after intrapartum asphyxia.

Methods: Retrospective cohort study in term infants with post-asphyxial hypoxic ischaemic encephalopathy (HIE) born between 1985 and 1995. Severe and moderate hyperoxaemia were defined as $\mathrm{PaO}_{2}>26.6$ and $\mathrm{PaO}_{2}>13.3 \mathrm{kPa}\left(200\right.$ and $100 \mathrm{~mm} \mathrm{Hg}$ ). Severe and moderate hypocapnia were defined as $\mathrm{PaCO}_{2}$ $<2.6$ and $\mathrm{PaCO}_{2}<3.3 \mathrm{kPa}(20$ and $25 \mathrm{~mm} \mathrm{Hg}$ ). Adverse outcome ascertained by age 24 months was defined as death, severe cerebral palsy, or any cerebral palsy with blindness, deafness, or developmental delay. With outcome as the dependent variable, multivariate analyses were performed including hyperoxaemic and hypocapnic variables, and factors adjusted for initial disease severity.

Results: Of 244 infants, 218 had known outcomes, 127 of which were adverse (64 deaths, 63 neurodevelopmental deficits). Multivariate analyses showed an association between adverse outcome and episodes of severe hyperoxaemia (odds ratio (OR) 3.85, 95\% confidence interval (CI) 1.67 to 8.88 , $\mathrm{p}=0.002$ ), and severe hypocapnia (OR 2.34, $95 \% \mathrm{Cl} 1.02$ to $5.37, \mathrm{p}=0.044)$. The risk of adverse outcome was highest in infants who had both severe hyperoxaemia and severe hypocapnia (OR 4.56, $95 \% \mathrm{Cl} 1.4$ to $14.9, \mathrm{p}=0.012)$.

Conclusions: Severe hyperoxaemia and severe hypocapnia were associated with adverse outcome in infants with post-asphyxial HIE. During the first hours of life, oxygen supplementation and ventilation should be rigorously controlled.
\end{abstract}

$\mathrm{n}$ resuscitating severely asphyxiated infants after birth, it is difficult to immediately achieve and maintain the correct balance between hyperoxaemia and hypoxaemia, and between hypocapnia and hypercapnia. Oxygen supplementation and/or ventilation may be inadequate or excessive because of variable postnatal cardiorespiratory adaptation and responses to therapeutic interventions, difficulty in monitoring and interpreting blood gas measurements, and inability to implement corrective action rapidly. More respiratory support than required results in hyperoxaemia or hypocapnia, which may increase the risk of brain insult and injury. ${ }^{1-4}$

The adverse effects of hyperoxaemia on the brain are mediated primarily by increased toxic free oxygen radicals. ${ }^{12}$ Hyperoxaemia may be particularly hazardous to the brain during the period of reperfusion after severe asphyxia. ${ }^{25}$ In animal models of asphyxia, oxygen supplementation increases free oxygen radical formation, ${ }^{67}$ decreases cerebral perfusion, ${ }^{8}$ and adversely affects short term neurological outcome. ${ }^{9}$ Similar effects were observed in human neonates exposed to oxygen during resuscitation. ${ }^{1-3}{ }^{10}$ Hypocapnia in animals is known to decrease cerebral blood flow, ${ }^{11-13}$ but does not occur consistently in human neonates. ${ }^{14}$

Infants with post-asphyxial hypoxic ischaemic encephalopathy (HIE) often have episodes of hyperoxaemia and/or hypocapnia between birth and transfer to our care. This provided data for analysis of associations between hyperoxaemia and/or hypocapnia in the first postnatal hours and adverse outcomes. Our objective was to determine if hyperoxaemia and/or hypocapnia add to the risk of brain injury after intrapartum asphyxia.

\section{METHODS \\ Population}

The subjects of this retrospective study were admitted after intrapartum asphyxia between 1985 and 1995 to the neonatal intensive care unit (NICU) at the Hospital for Sick Children, Toronto, which is the regional tertiary referral centre for about 55000 annual term births. The reasons for admission were continued ventilator dependency and/or HIE.

\section{Eligibility criteria}

The following four mandatory inclusion criteria were modifications of the statement of the Society of Obstetricians and Gynecologists of Canada. ${ }^{15}$

(1) One or more of the following:

(a) Five minute Apgar score less than 5

(b) Metabolic acidosis (cord arterial blood base deficit $\geqslant$ $16 \mathrm{mmol} / \mathrm{l}$ )

(c) Delayed onset of respiration for five or more minutes

(d) Birth by emergency caesarean section for fetal distress.

(2) Need for mechanical ventilation immediately after delivery.

Abbreviations: $\mathrm{Cl}$, confidence interval; $\mathrm{FiO}_{2}$, fraction of inspired oxygen; $\mathrm{HIE}$, hypoxic ischaemic encephalopathy; $\mathrm{NICU}$, neonatal intensive care unit; $\mathrm{OR}$, odds ratio; $\mathrm{PaO}_{2}$, partial arterial oxygen pressure; $\mathrm{PaCO}_{2}$, partial arterial carbon dioxide pressure 
(3) Evidence of encephalopathy including altered state of consciousness and/or seizures.

(4) Multiorgan involvement (encephalopathy and at least one other organ or system involved).

\section{Exclusion criteria}

Infants were excluded for preterm delivery ( $<37$ weeks gestation), any congenital abnormality (including brain abnormalities considered to predate the intrapartum period), inborn errors of metabolism, congenital infections, septic or haemorrhagic shock, meningitis, severe meconium aspiration syndrome, ${ }^{16}$ or cranial birth trauma. Infants with antenatal compromise (loss of fetal movements for more than 24 hours, lack of fetal heart variability at time of admission of mother to hospital, or oligohydramnios) were excluded because brain damage may have occurred antepartum. ${ }^{17}{ }^{18}$

\section{Search for subjects and data collection}

Infants with HIE were found by searching the NICU and hospital health records databases. Data related to maternal and obstetric history, pregnancy, parturition, transport, resuscitation, and neonatal course were collected retrospectively from patient records. The results of blood gas analyses and ventilation variables were collected for the first four hours after birth. Outcome data were determined from the records of neonatal follow up and neurology clinics and from readmissions to the hospital. Outcome data available at 1 year of age included assessment of motor, visual, and auditory function. Infants with questionable developmental delay had Bayley scores measured at 21-24 months of age. Where follow up documentation was incomplete, the infant's family doctor or paediatrician was contacted by telephone, and, if considered appropriate, the infant's family was requested by mail to participate in a telephone interview. Non-responders were considered lost to follow up. The Research Ethics Board of The Hospital for Sick Children approved the study.

\section{Definitions of exposure to hyperoxaemia and hypocapnia}

Two levels of hyperoxaemia and hypocapnia were defined as follows. Moderate hyperoxaemia was defined as a partial arterial pressure of oxygen $\left(\mathrm{PaO}_{2}\right)>13.3 \mathrm{kPa}(100 \mathrm{~mm} \mathrm{Hg})$, and severe hyperoxaemia as $\mathrm{PaO}_{2} \geqslant 26.6 \mathrm{kPa}(200 \mathrm{~mm} \mathrm{Hg})$. Moderate hypocapnia was defined as a partial arterial pressure of carbon dioxide $\left(\mathrm{PaCO}_{2}\right)<3.3 \mathrm{kPa}(25 \mathrm{~mm} \mathrm{Hg})$ and severe hypocapnia as $\mathrm{PaCO}_{2}<2.6 \mathrm{kPa}(20 \mathrm{~mm} \mathrm{Hg}$ ) (the former has been selected for study by others). ${ }^{19}$ These definitions were chosen before statistical analysis.

\section{Definitions of outcomes}

Adverse outcome was defined as death or severe neurodevelopmental disability in survivors as ascertained by one or more of the following:

(1) Severe cerebral palsy, defined as severe impairment of daily activities associated with hypertonia or hyperreflexia, diagnosed by 12 months of age.

(2) Mild to moderate cerebral palsy and developmental delay at 12 months of age, both confirmed at 21-24 months of age. Mild to moderate cerebral palsy was defined by impairment of daily activities associated with hypertonia or hyper-reflexia as determined by a qualified caregiver in the follow up or neurology clinics. Developmental delay was determined by a Bayley score lower than 2 SD below the mean for age, between 21 and 24 months of age.
(3) Any cerebral palsy with cortical blindness or sensorineural deafness attributable to asphyxia, diagnosed by 12 months of age.

\section{Statistical analysis}

Data were described using summary measures including mean, median, and standard deviation. Univariate analyses relating hyperoxaemic and hypocarbic predictor variables and the number of blood gas analyses per patient to outcome were performed using the $\chi^{2}$ test and simple univariate logistic regression modelling. For covariates measured on an ordinal scale, association with outcome was determined using the Cochran-Armitage trend test. To minimise the effect of the initial resuscitation, data obtained during the first 20 minutes of life were not included in the analyses. Analysis of data was limited to those available up to 120 minutes of age because of the time dependent variables used to adjust for initial disease severity.

Univariate analyses of association were examined between (a) occurrence of hyperoxaemia (moderate or severe) in any arterial blood gas during the first 20-120 minutes of life and outcome; $(b)$ occurrence of hypocapnia (moderate or severe) in any arterial blood gas during the first 20-120 minutes of life and outcome; (c) exposure to severe hyperoxaemia and/or severe hypocapnia (a combined exposure variable ${ }^{20}$ with three categories of exposure: to neither severe hyperoxaemia or hypocapnia, to either, or to both) and outcome. Risk factor variables associated with adverse outcome at $p<0.1$ in univariate analyses were included in the multivariate analyses.

Multivariate logistic regression analyses using forward stepwise selection were conducted, controlling for disease severity. Three measures of initial disease severity from previous studies ${ }^{21-23}$ were entered into multivariate analyses: five minute Apgar scores, time to establish a sustained breathing pattern, and first available base deficit measured as the number of standard deviations from the mean normal value. It was planned to enter also the number of blood gas measurements if the $p$ value of this variable was under 0.1 in univariate analysis. In multivariate analyses, $\mathrm{p}<0.05$ was deemed significant. Statistical analysis was performed using the SAS software (version 8.02; SAS Institute Inc, Cary, North Carolina, USA).

\section{RESULTS}

During 1985-1995, 244 term infants eligible for the study were admitted to the NICU. The mean (SD) gestational age and birth weight of the infants were 40.0 (1.6) weeks and 3450 (520) g. Boys made up 60\% of the infants. Severe metabolic acidosis occurred in 135 of 236 infants, and the mean (SD) first available base deficit was 17.2 (7.5). Delayed onset of respiration for five or more minutes was observed in 146 of 209 infants, with a mean time to establish regular breathing of 30.3 (45.9) minutes. A five minute Apgar score $\leqslant 4$ was noted in 145 of 236 infants; the mean five minute Apgar score was 3.7 (2.3). Outcome data were obtained for 218 of 244 infants $(89 \%)$, by evaluation in the hospital of 207 patients and by telephone interview for 11 . By 2 years of age, 127 infants (58\%) had adverse outcomes. Of the 127 infants, 64 infants died and 63 had severe neurodevelopmental impairment ( 56 had severe cerebral palsy, seven had mild or moderate cerebral palsy together with a Bayley score $>2$ SD below the mean at 21-24 months). Detailed infant characteristics and outcomes have been previously reported. ${ }^{22}$ Table 1 shows ventilation rates and oxygen supplementation during the first 2 hours of life. Arterial blood gas measurements were available for 173 infants between 20 minutes and 2 hours of age. Episodes of moderate and 
Table 1 Ventilation rates and oxygen supplementation administered between 30 and 120 minutes of age to term infants with birth asphyxia $(n=173)$

\begin{tabular}{llllll}
\hline & \multicolumn{2}{l}{ Ventilation rate (breaths/min) } & & \multicolumn{2}{l}{$\mathrm{FiO}_{2}(\%)$} \\
\cline { 2 - 3 } Time $(\mathbf{m i n})$ & Mean (SD) & Median & & Mean (SD) & Median \\
\hline 30 & $51(23)$ & 60 & $0.94(0.17)$ & 1.00 \\
60 & $41(27)$ & 60 & $0.85(0.24)$ & 1.00 \\
120 & $38(30)$ & 45 & $0.76(0.29)$ & 0.96 \\
\hline
\end{tabular}

Non-ventilated infants were considered to have a zero rate of ventilation.

severe hyperoxaemia were identified in $118(68 \%)$ and 64 $(37 \%)$ infants. Episodes of moderate and severe hypocapnia occurred in $92(53 \%)$ and $59(34 \%)$ infants. Thirty seven infants $(21 \%)$ had both severe hyperoxaemia and severe hypocapnia.

\section{Univariate analyses}

During the first 20-120 minutes of life, any episode of severe hyperoxaemia, moderate hypocapnia, or severe hypocapnia was associated with adverse outcome. The adverse outcome rate of infants exposed to both severe hyperoxaemia and severe hypocapnia was $80 \%$, to either $71 \%$, and to neither $49 \%$, suggesting a dose-response relation (Cochran-Armitage trend test $\mathrm{p}=0.02$ ). A near association was found between the number of blood gas measurements and outcome $(\mathrm{p}=$ $0.07)$.

\section{Multivariate analyses}

Of 218 infants with complete follow up, 139 had blood gas analyses between 20 and 120 minutes of age, and were available for multivariate analyses. Age at onset of regular breathing, five minute Apgar score, base deficit, and the number of blood gas analyses per subject were entered into the multivariate analyses to control for severity of illness. The variable of interest in each analysis was the hyperoxaemic or hypocarbic variable found in univariate analyses to be associated with outcome, namely severe hyperoxaemia, severe hypocapnia, and moderate hypocapnia. During the first 20-120 minutes, any occurrence of severe hyperoxaemia (OR $3.85,95 \%$ CI 1.67 to $8.88, \mathrm{p}=0.002$ ) and any occurrence of severe hypocapnia (OR 2.34, 95\% CI 1.02 to $5.37, \mathrm{p}=$ 0.044 ) were significantly associated with adverse outcome The four variables entered to correct for disease severity did not remain in the model with severe hyperoxaemia; and only time of onset of regular breathing remained in the model

Table 2 Multivariate analysis of association of severe hyperoxaemia and/or severe hypocapnia with adverse outcome during the first 20-120 minutes of life in infants with birth asphyxia $(n=139)$, controlling for disease severity

\begin{tabular}{|c|c|c|c|}
\hline Variable & OR & $95 \% \mathrm{Cl}$ & p Value \\
\hline $\begin{array}{l}\text { Severe hyperoxaemia or severe } \\
\text { hypocapnia }\end{array}$ & 3.07 & 1.31 to 7.18 & 0.001 \\
\hline $\begin{array}{l}\text { Severe hyperoxaemia and severe } \\
\text { hypocapnia }\end{array}$ & 4.56 & 1.4 to 14.9 & 0.012 \\
\hline Time to regular breathing ${ }^{*}$ & 2.29 & 1.00 to 5.22 & 0.049 \\
\hline Number of blood gases & - & - & NS \\
\hline Base deficitt & - & - & NS \\
\hline 5 min Apgar score & - & - & NS \\
\hline \multicolumn{4}{|c|}{$\begin{array}{l}\text { Severe hyperoxaemia and severe hypocapnia were defined as } \mathrm{PaO}_{2} \\
>26.6 \mathrm{kPa}(200 \mathrm{~mm} \mathrm{Hg}) \text { and } \mathrm{PaCO}_{2}<2.6 \mathrm{kPa}(20 \mathrm{~mm} \mathrm{Hg}) \\
\text { respectively. } \\
{ }^{*} \text { Time from birth in minutes to establishment of a regular breathing } \\
\text { pattern. } \\
\text { TFirst available base deficit, measured in } \mathrm{SDs} \text { from mean normal values. }\end{array}$} \\
\hline
\end{tabular}

with severe hypocapnia (OR 4.35, 95\% CI 1.06 to 5.23, p = 0.049 ). No association was found between adverse outcome and moderate hypocapnia. Table 2 shows analyses for possible interactions between severe hyperoxaemia and severe hypocapnia. The strongest association with adverse outcome was for infants with both severe hyperoxaemia and severe hypocapnia (OR 4.56, 95\% CI 1.4 to $14.9, \mathrm{p}=0.012$ ).

\section{DISCUSSION}

Adjusting for the severity of birth asphyxia, we found that peak $\mathrm{PaO}_{2}$ values exceeding $26.6 \mathrm{kPa}(200 \mathrm{~mm} \mathrm{Hg})$ and trough $\mathrm{PaCO}_{2}$ values of $2.6 \mathrm{kPa}(20 \mathrm{~mm} \mathrm{Hg})$ or lower during the first 20 to 120 minutes of life were associated with death or adverse neurodevelopmental outcome. The risk of adverse outcome was greatest in subjects who had both.

Mechanisms of oxygen induced brain injury in animals are well established. ${ }^{6-9}$ Human studies of exposure to $100 \%$ oxygen have focused on its use during the immediate resuscitation. ${ }^{324-26}$ Exposure to $100 \%$ oxygen increased biochemical markers of oxidative stress to 15 minutes of life. ${ }^{3}$ Clinically, onset of respiration was delayed and resuscitation prolonged, but mortality was not increased. ${ }^{24-26}$ In our study $\mathrm{PaO}_{2}$ values exceeding $26.6 \mathrm{kPa}(200 \mathrm{~mm} \mathrm{Hg}$ ) were associated with adverse outcome, but lower exposure levels were not. This is probably a dose effect.

Hypocapnia decreases cerebral blood flow in animal models, ${ }^{12}{ }^{13}$ but not consistently in human neonates. ${ }^{14}$ Hypocapnia, by decreasing cerebral blood flow, may increase the risk of injury in ischaemic cerebral disease states. In preterm infants, hypocapnia was found to be associated with increased risk of periventricular leucomalacia ${ }^{19}$ and cerebral palsy. ${ }^{27}$ Hypocapnia in animal models interacts adversely with hyperoxaemia with respect to cerebral perfusion and metabolism..$^{132}$ We similarly found evidence of a possible additive effect of severe hypocapnia and severe hyperoxaemia. In many post-asphyxial infants, cardiorespiratory function improves rapidly, outstripping weaning and resulting in unintentional hyperoxaemia and/or hypocapnia. This is evidenced in this study by the high incidence of hyperoxaemia and hypocapnia, and by the relatively high ventilation rates and $\mathrm{FiO}_{2}$.

Current North American neonatal resuscitation guidelines recommend the use of $100 \%$ inspired oxygen, ${ }^{29}$ whereas British guidelines suggest that "it may be more appropriate to use an inspired oxygen concentration of $40 \%$ initially and increase this if required". ${ }^{30}$ Recommended initial ventilation rates vary between $40-60$ breaths $/ \mathrm{min}^{29}$ and $30-40$ breaths/ min. ${ }^{30}$ Although the results of this study may not be applicable to resuscitation of the asphyxiated neonate in the first 20 minutes after birth, the initial choice of respiratory support may influence the duration of weaning for a few hours. Even with closely monitored ventilation, hypocapnia and hyperoxaemia occurred in 38\% and $25 \%$ of preterm infants during transfer to the NICU. ${ }^{4}$ Neonatal resuscitation guidelines could draw more attention to rigorous post-resuscitation monitoring and appropriate 
responses to changes in $\mathrm{PaO}_{2}$ and $\mathrm{PaCO}_{2}$, to avoid the potential risks of prolonged hyperoxaemia and hypocapnia.

Randomised controlled trials are the preferred method to answer well defined therapeutic questions; however, to answer our question, this study design would be questionable. Once vital signs are established, oxygen therapy and ventilation are adjusted to the individual's needs. In the light of the known adverse effects of hyperoxaemia and hypocapnia, it would not be ethical to conduct a trial involving prolonged exposure for up to two hours to $100 \%$ oxygen or a higher ventilation rate than required by the individual.

Retrospective studies have well recognised limitations. In our study these were mitigated to some extent by consistency throughout the study period of resuscitation guidelines including use of $100 \%$ oxygen as standard practice, referral patterns, management by our transport team, and follow up assessment. The inclusion of subjects born as long as 19 years ago, which was necessary to collect an adequate sample of patients, raises the question of the applicability of our results to contemporary patients. Only minor changes in the management of post-asphyxial encephalopathy occurred during the study period and no policy changes were made in our unit regarding the provision or withdrawal of life support.

Selection bias existed against transfer of patients with mild asphyxia and Sarnat stage I HIE. During the study period, treatment at referring hospitals was aggressive, and life support was withdrawn only after patient transfer to our care. Thus few patients died in their hospitals of birth, and the majority of the most severely ill patients were included in our study. Moreover, the $\mathrm{PaO}_{2}$ and $\mathrm{PaCO}_{2}$ values played no role in decisions to withdraw life support, whereas the time to establish regular breathing and the first base deficit values used to control for severity of illness may have been considered in decision making because of their recognised prognostic value. ${ }^{21-23}$

We found that, in infants with severe asphyxia, severe hyperoxaemia was associated with adverse long term outcome, which increased with coexisting severe hypocapnia. Although we have shown association but not causation, we consider that our findings have clinical implications. Normoxia and normocapnia would appear to be proper therapeutic goals, attainable with closer monitoring and individualised oxygen supplementation and ventilation.

\section{Authors' affiliations}

G Klinger, Division of Neonatology, Hospital for Sick Children, Toronto, Ontario, Canada

J Beyene, P Shah, Department of Paediatrics, Mount Sinai Hospital, Toronto

J Beyene, Research Institute, Hospital for Sick Children, Toronto

G Klinger, J Beyene, P Shah, M Perlman, University of Toronto

Competing interests: MP acts as a consultant and provides expert written and oral testimony on behalf of plaintiffs, physicians, and hospitals related to medical malpractice claims for compensation. The claims are made by children with brain injuries associated with intrapartum asphyxia. He has not provided evidence, and does not intend to testify, that hyperoxia and/or hypocarbia contributed to the cause of brain and other injuries of term infants with post-intrapartum asphyxial encephalopathy and cerebral palsy.

\section{REFERENCES}

1 Volpe JJ. Hypoxic-ischemic encephalopathy: clinical aspects. In: Neurology of the newborn. 4th ed. Philadelphia: WB Saunders Company, 2001:331-94.
2 Saugstad OD. Resuscitation of newborn infants with room air or oxygen. Semin Neonatol 2001;6:233-9.

3 Vento $M$, Asensi M, Sastre J, et al. Oxidative stress in asphyxiated term infants resuscitated with 100\% oxygen. J Pediatr 2003;142:240-6.

4 Tracy M, Downe L, Holberton J. How safe is intermitttent positive pressure ventilation in preterm babies ventilated from delivery to newborn intensive care unit? Arch Dis Child Fetal Neonatal Ed 2004;89:F184-7.

5 Saugstad OD. Resuscitation of the asphyxic newborn infant. New insight leads to new therapeutic possibilities. Biol Neonate $2001 ; 79: 258-60$.

6 Capani F, Loidl CF, Aguirre F, et al. Changes in reactive oxygen species (ROS) production in rat brain during global perinatal asphyxia: an ESR study. Brain Res 2001:914:204-7.

7 Lievre V, Becuwe $P$, Bianchi A, et al. Intracellular generation of free radicals and modifications of detoxifying enzymes in cultured neurons from the developing rat forebrain in response to transient hypoxia. Neuroscience 2001;105:287-97.

8 Rosenberg AA, Murdaugh E, White CW. The role of oxygen free radicals in postasphyxia cerebral hypoperfusion in newborn lambs. Pediatr Res 1989:26:215-19.

9 Temesvari P, Karg E, Bodi I, et al. Impaired early neurologic outcome in newborn piglets reoxygenated with 100 percent oxygen compared with room air after pneumothorax-induced asphyxia. Pediatr Res 2001;49:812-19.

10 Lundstrom KE, Pryds O, Greisen G. Oxygen at birth and prolonged cerebral vasoconstriction in preterm infants. Arch Dis Child Fetal Neonatal Ed 1995;73:F81-6.

11 Rosenberg AA. Response of the cerebral circulation to hypocarbia in postasphyxia newborn lambs. Pediatr Res 1992:32:537-41.

12 Koons AH, Wurtzel D, Metcalf JM, et al. Cerebral blood flow measurements in the newborn dog. Biol Neonate 1993;63:120-8.

13 Vannucci RC, Bruchlacher RM, Vannucci SJ. Effect of carbon dioxide on cerebral metabolism during hypoxia-ischemia in the immature rat. Pediatr Res 1997:42:24-9.

14 Pryds O, Greisen G, Lou H, et al. Heterogeneity of cerebral vasoreactivity in preterm infants supported by mechanical ventilation. J Pediatr 1989;115:638-45

15 Policy Statement. Task force on cerebral palsy and neonatal asphyxia (part 1). J SOGC 1996;18:1267-79.

16 Gillan JE, Pape KE, Cutz E. Association of changes in Bombesin immunoreactive neuroendocrine cells in lungs of newborn infants with persistent fetal circulation and brainstem damage due to birth asphyxia. Pediatr Res 1986;20:828-33.

17 Myers RE. Two patterns of perinatal brain damage and their conditions of occurrence. Am J Obstet Gynecol 1972;112:246-76.

18 Phelan JP, Ahn MO. Perinatal observations in forty-eight neurologically impaired term infants. Am J Obstet Gynecol 1994;171:424-31.

19 Okumura A, Hayakawa F, Kato T, et al. Hypocarbia in preterm infants with periventricular leukomalacia: the relation between hypocarbia and mechanical ventilation. Pediatrics 2001;107:469-75.

20 Agresti A. Categorical data analysis. New York: Wiley, 1990.

21 Ekert P, Perlman M, Steinlin M, et al. Predicting outcome of severe perinatal asphyxia in term infants in the first 4 hours of life. J Pediatr 1997;131:613-17.

22 Shah PS, Raju NV, Beyene J, et al. Recovery of metabolic acidosis in term infants with postasphyxial hypoxic-ischemic encephalopathy. Acta Paediat 2003:92:941-7.

23 Peliowski A, Finer NN. Birth asphyxia in the term infant. In: Sinclair JS, Bracken MB, eds. Effective care of the newborn infant. Oxford: Oxford University Press, 1992:249-80

24 Ramii S, Ahuja S, Thirupuram S, et al. Resuscitation of asphyxic newborn infants with room air or 100 percent oxygen. Pediatr Res 1993;34:809-12

25 Saugstad OD, Rootwelt T, Aalen O. Resuscitation of asphyxiated newborn infants with room air or oxygen: an international controlled trial: the Resair 2 study. Pediatrics 1998;102:e1.

26 Ramii S, Rasaily R, Mishra PK, et al. Resuscitation of asphyxiated newborn with room air or $100 \%$ oxygen at birth: a multicentric clinical trial. Indian Pediatr 2003;40:510-17.

27 Graziani L, Spitzer AR, Mitchell DG. Mechanical ventilation in preterm infants: neurosonographic and developmental studies. Pediatrics 1992;90:515-22.

28 Vannucci RC, Towfighi J, Heitian DF, et al. Carbon dioxide protects the perinatal brain from hypoxia-ischemic damage: an experimental study in the immature rat. Pediatrics 1995;95:868-74.

29 Contributors and Reviewers for the Neonatal Resuscitation Guidelines. International guidelines for neonatal resuscitation: an excerpt from the guidelines 2000 for cardiopulmonary resuscitation and emergency cardiovascular care: International Consensus on Science. Pediatrics 2000; 106:e29

30 Royal College of Paediatrics and Child Health and Royal College of Obstetricians and Gynaecologists. Resuscitation of babies at birth. London: BMJ Publishing Group, 1997. 